\title{
Subjektive Einschätzung der Patienten als Studienendpunkt
}

\author{
Der Einfluss medizinischer Therapien auf die Lebensqualität der Patienten \\ wird zunehmend als bedeutsamer Faktor im Management von Krebserkran- \\ kungen erkannt. Daher beinhalten immer mehr Studien einen Endpunkt, \\ dessen Messgrößen von den Patienten beurteilt wurden.
}

wie zur Lokalisation des Tumors durchgeführt. Von den Patienten beurteilte Messgrößen wurden in 557 randomisierten, kontrollierten Studien mit insgesamt 254.677 Patienten untersucht. Am häufigsten wurden diese in Studien zu Brustkrebs $(\mathrm{n}=123)$, Lungenkrebs $(\mathrm{n}=85)$ und kolorektalen Karzinomen $(\mathrm{n}=66)$ angewendet.

In $24 \%$ der Studien waren die von den Patienten beurteilten Messgrößen primäre Endpunkte, in $76 \%$ der Studien sekundäre Endpunkte. In der CONSORT PRO Extension werden 6 Kriterien gefordert, die 2 meist berichteten waren: In $81 \%$ der Studien wurden PROs im Abstract beschrieben, und in $76 \%$ der Studien wurden validierte $\mathrm{PRO}$-Instrumente verwendet. Die übrigen 4 Aspekte wurden in weniger als $50 \%$ der Studien angewendet. Weniger als $5 \%$ der Studien berücksichtigten alle 6 Kriterien der CONSORT PRO Extension.

Das Dokumentationsniveau war in Studien, in denen ein PRO als primärer Endpunkt definiert war, signifikant höher. Das Vorliegen eines zusätzlichen Berichts war der einzige statistisch signifikante Faktor, der mit einem höheren Niveau der PRO-Dokumentation assoziiert war. Dies galt sowohl für Studien mit PRO als primäre Endpunkte sowie für Studien mit PRO als sekundäre Endpunkte (jeweils $\mathrm{p}<0,001$ ).

Fazit: Die Implementierung der Kriterien der CONSORT PRO Extension ist bei allen Krebsarten gleich wichtig. Seine Anwendung kann dazu beitragen, Studien mit robustem PRO-Design zu identifizieren.

Judith Neumaier

Efficace F et al. Quality of Patient-Reported Outcome Reporting Across Cancer Randomized Controlled Trials According to the CONSORT Patient-Reported Outcome Extension: A Pooled Analysis of 557 Trials. Cancer. 2015;121(18):3335-42.

\section{Die meisten Patienten mit Darmkrebs- und Lungenkrebs glauben an Heilung durch OP}

\begin{abstract}
Aktuelle Daten weisen darauf hin, dass Krebspatienten das Ziel bestimmter Therapien nicht verstehen. In einer Studie wurde untersucht, was Patienten mit einem Tumor in Lunge oder Kolorektum von einer Operation erwarten.
\end{abstract}

nsgesamt 3.954 Patienten, die an der
CanCORS-Studie teilgenommen und
sich einer krebsbedingten Operation der
Lunge (30,3\%) oder des Kolorektums
$(69,7 \%)$ unterzogen hatten, wurden in
die Studie aufgenommen. Innerhalb von
vier bis sechs Monaten nach Diagnose
wurden Sie per E-Mail zu ihren Einstel-
lungen, Behandlungszielen, Therapieent-
scheidungen und Lebensqualität befragt.
Etwa $80 \%$ der Patienten mit Lungen-
und $89,7 \%$ mit kolorektalem Karzinom
gingen davon aus, dass die Operation
ihre Krebserkrankung wahrscheinlich
heilen würde, wobei die Hoffnung mit
zunehmendem Tumorstadium sank.
Dennoch glaubten immer noch 57,4 bzw. $79,8 \%$ der Patienten mit einem Tumor im Stadium IV, durch die Operation wahrscheinlich geheilt zu werden.

In multivariaten Analysen lag die Odds Ratio (OR) für die Annahme einer kurativen Intention bei Patienten mit kolorektalem Karzinom höher als bei denen mit Lungenkrebs (OR 2,27). Frauen (OR 0,79), nicht verheiratete Patienten (OR $0,78)$, solche in fortgeschrittenen Tumorstadien (mit Metastasen vs. im Stadium I/II: OR 0,28) und Patienten mit 3 oder mehr Komorbiditäten $(\mathrm{OR} 0,78)$ glaubten seltener an Heilung durch Operation. Bildungsniveau, körperliche Funktion und
Versicherungsstatus waren nicht mit dem Glauben an eine Heilung assoziiert.

Nur etwa die Hälfte $(55,6 \%)$ der Patienten beurteilte die Kommunikation mit ihrem Arzt als optimal. Sie gingen im Vergleich zu den übrigen häufiger (OR 1,89 ) davon aus, dass die Operation kurativ sein würde. Darüber hinaus gingen Patienten, die keinen Einfluss auf die Therapieentscheidung hatten, seltener $(\mathrm{OR} 0,56)$ von einer Heilung durch die Operation aus als Patienten, die selbst oder über ihre Familie an der Therapieentscheidung beteiligt waren.

Fazit: Ein Großteil der Patienten mit Lungen- oder kolorektalem Karzinom geht davon aus, dass eine Operation kurativ ist. Künftig sollte man die Arzt-PatientenKommunikation bezüglich der Ziele der Behandlung optimieren. Judith Neumaier

Kim Y et al. Patient Perceptions Regarding the Likelihood of Cure After Surgical Resection of Lung and Colorectal Cancer. Cancer. 2015;121(20):3564-73. 